\title{
Ask the Experts: how to curb antibiotic resistance and plug the antibiotics gap?
}

\author{
First draft submitted: 9 April 2016; Accepted for publication: 6 May 2016; \\ Published online: 21 June 2016
}

The mid-20th century was witness to the most prolific antibiotic discovery era yet, however, in the years since, interest in the field has waned and there is now a dramatic shortage of new classes of antibiotics under development. With the rise of antibacterial drug resistance, there has been much debate across the scientific community regarding how best to bolster the antibacterial drug pipeline. While there is an increasing focus on the search for new scaffolds and drug targets, efforts are also being channelled towards more short-term solutions, such as rejuvenating current antibiotics and drug repurposing. In addition, leaders in the field are calling for an urgent shake-up with regards to funding, regulatory restrictions, intellectual property and pricing models for the sale of new drugs, in order to help stimulate the discovery and development of novel antibacterial agents. Future Medicinal Chemistry invited a selection of experts to express their views on the major hurdles in plugging the antibiotic gap, possible approaches to curbing a potential antibiotic crisis, and how they perceive the field of antibacterial drug discovery will develop over the coming years. Their enlightening responses provide an insight into the complexities of this issue and a snapshot of the strategies that are being put into place to help catalyze antibacterial drug research.

\section{Laura Piddock}

Q What do you believe is the major hurdle in addressing antibiotic resistance?

The major hurdle is lack of funding globally. Therefore, this means that each aspect of addressing the crisis is carried out piecemeal, and some countries are able to do more than others. However, there are some aspects that are country specific, such as infection prevention and control. These have to be addressed to prevent the onward transmission and dissemination of antibiotic resistance genes and bacteria. So addressing public health and ensuring that everyone has access to clean water, and that their environment is not contaminated by sewage is paramount. This also includes installing closed sewers in many countries. Breaking the transmission chain is very important. This is because even if we are able to make new drugs or alternative treatments quickly, it is likely that there will always be bacteria that are able to evade these or develop resistance. Therefore, minimizing resistance development and transmission is a key global priority.

Q After a hugely prolific antibiotic discovery period between 1940 \& 1962, there is now a dramatic shortage of new classes of antibiotics under development. What do you believe is the reason for this? The primary reason that there has been a dramatic shortage of new classes of antibiotics under development is because this discipline was primarily the domain of pharmaceutical companies. As it became clear that finding new antibiotics was challenging and the return of investment was poor compared with other therapeutic areas, fewer and fewer people were involved in this activity. One of the key reasons there was a dramatic shortage of new classes is that there was a perception in the 1960s and into the 1970s that the 'war' against bacterial infections had been won and as there were many different antibiotics that no new drugs would be needed. It had also become clear that the changing nature of the problem, in other words, there were more infections by Gram-negative bacteria than previously, meant that new drugs and therefore new classes of drugs were required to tackle this challenge. This has proved extremely difficult due to the nature of the Gram-negative bacterial cell envelope that only allows a small number of drug-like molecules into it, and the presence of multidrug efflux pumps that export many of these drugs out. Therefore, obtaining inhibitory or cidal concentrations of antibiotic mol-

\section{Future \\ Medicinal Chemistry}

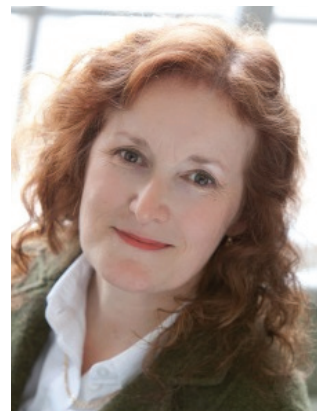

Laura Piddock

Institute of Microbiology and Infection University of Birmingham, Birmingham, UK

I.j.v.piddock@bham.ac.uk

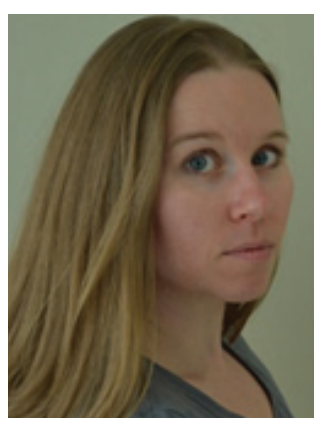

Sylvie Garneau-Tsodikova Department of Pharmaceutical Sciences, University of Kentucky, KY, USA sylviegtsodikova@uky.edu

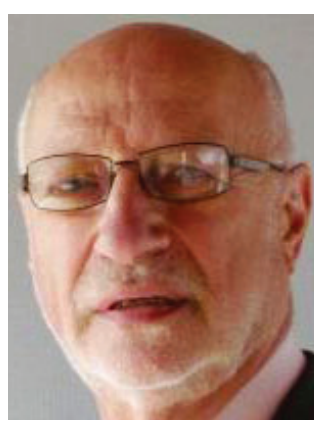

Colin Garner

Antibiotic Research UK, York, UK colin.garner@antibioticresearch.org.uk

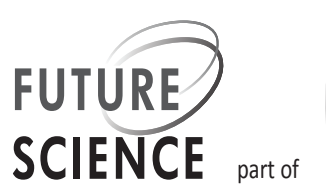


ecules in Gram-negative bacteria remains a technical challenge.

Q Are there any alternative approaches that you believe may yield more promising results, but are currently being overlooked in the discovery of new antimicrobials?

I do not think that those involved in discovery are overlooking alternative approaches. It is clear that for the last 10-15 years, discoveries have been made outside of large pharmaceutical companies and been in the domain of academia, research institutes and spin-off companies and small medium enterprises. There is still much to be done and despite this area moving out of companies, traditional national funding agencies have not been quick to support research in this area. One issue that remains today for the development of alternative approaches, is whether they would be used by healthcare professionals and what the regulatory pathway will be. This is the challenge for bacteriophage preparations to which bacteria can become resistant quickly. Therefore, cocktails of phages are being proposed, but there is no regulatory pathway specific for phages and so it is likely they will be licensed as a medication and follow the same regulatory pathway as antibiotic drugs. This is clearly an issue for those developing these approaches.

Q Taking into account that the majority of antibiotics that are in use today do not strictly adhere to the Lipinski rule of five, do you feel that classically used 'rules of thumb', such as this, should be avoided in the discovery \& design of new antibiotics?

It is very clear that the Lipinski rules are not really suitable for antibiotic drugs. It is hoped that there will be rules governing which chemical properties are desirable in an antibiotic molecule that will indicate whether it could be developed into a drug for patients. A recent publication (Progress Against Escherichia coli With The Oxazolidinone Class of Antibacterials: Test Case for a General Approach To Improving Whole-Cell GramNegative Activity [1]) has suggested how one class of antibiotic can be improved such that they enter into Gramnegative bacteria and are less susceptible to multidrug efflux pumps.

Q It has been argued that if penicillin was discovered today, it would never have made it to the market due to regulatory restrictions. With this in mind, do you feel that current regulations stifle the development of novel antibiotics?

A few years ago, the answer to this question would have been yes. However, the European Medicines Agency and the US FDA have worked hard with companies to develop guidelines for the appropriate clinical trials and endpoints for studies that will make the approvals process for antibiotics much clearer. Therefore, I do not think that the current regulations are stifling development today.

Q Bearing in mind that the issue of antimicrobial resistance is more acute in developing countries, how can it be ensured that new antimicrobials are priced at a level that is both commercially attractive to pharmaceutical companies $\&$ is also accessible to the developing world?

This is a very important point and is being investigated in the EU IMI-funded project, DRIVE-AB. It is also important that various pricing models are proposed to encourage pharmaceutical companies to remain or return to antibacterial drug development, but also that there is equitable access to effective drugs throughout the world. At the current time, it is clear that there will be several economic models that will be recommended as the price of drugs clearly influences access in some countries. However, these issues have been tackled for anti-HIV drugs and the DRIVE-AB project is looking to make its recommendations no later than September 2017.

Q To what extent do you believe governments worldwide can help stem the rise of antibiotic resistance \& stimulate research toward new drugs? I believe governments globally should continue to work together and help put in place good public health systems in low-income countries that will help prevent transmission of drug-resistant bacteria. It is also important that the public play a role in preventing the increase in numbers of antibiotic-resistant infections. It is very important that public awareness initiatives such as Antibiotic Action (UK) [2] are able to translate scientific information into a format that is readily accessible and understood by the general public. For this reason Antibiotic Action is extending its work with other organizations, including working with producers of cartoons, live theater productions and a celebrity campaign with another organization about factory farming of pigs. We are also keen to encourage public awareness among children and young adults so that using antibiotics appropriately and responsibly becomes the norm.

Q The process of bringing new drugs from the bench to the bedside is on average 10-15 years. With this in mind, do you feel that investing in the discovery of innovative new drugs is a promising solution to the antibiotic crisis? It is important that we recognize that the discovery and development of antibiotic drugs is a relatively new field. If the scientific information available today about how to minimize resistance, and mechanisms of resistance, had been available 25 years ago then that would have 
guided discovery and development in a totally different direction. This is not a therapeutic area to be discarded, but one to build upon including with alternative treatments. New drugs will always be part of therapeutic options to treat infections as prescribers and patients are familiar and comfortable with this type of medication.

\section{Q How do you propose that we plug the} antibiotic gap in the short term?

There are new drugs that are currently either in the final stages of development or have recently been licensed. These will help plug the antibiotic gap, including for some Gram-negative bacterial infections in the short term. In the medium term, I believe that we must have good scientific and clinical evidence to use specific combinations of drugs rather than the ad hoc combinations that are sometimes chosen by prescribers.

Q Do you believe that the repurposing of drugs for other indications as antimicrobials provides a viable interim solution?

Repurposing of drugs is a very attractive option and has been commented upon by the AMR Review and others. However, evidence must be provided to show that drugs used for other therapeutic areas should be repurposed to use against bacterial infections or in combination with antibacterial drugs. Translating laboratory information on combinations needs to be tested properly in patients to determine whether these will be a viable solution.

Q Do you feel that improvements can be made to the way in which current antimicrobials are used? Improvements to the way in which current antimicrobials are used are imperative and will help solve the antimicrobial resistance crisis. This is why The British Society for Antimicrobial Chemotherapy (BSAC) launched a Massive Open Online Course (MOOC) in collaboration with the University of Dundee (Dundee, Scotland, UK) and Future Learn - Antimicrobial Stewardship: Managing Antibiotic Resistance [3]. Many across the world have already registered to take this course and educating prescribers is a key part of reducing use. This will prolong the effectiveness of currently available drugs.

\section{Q How do you anticipate the field of} antimicrobial drug discovery progressing over the next 5-10 years?

It is hard to predict how the field of drug discovery will progress in the next 5-10 years. This is because it is completely dependent on funding for this area which does not seem to be available at the same level as some of the other unmet medical needs such as Alzheimer's and obesity. This is why government involvement is so important because they can dictate the priorities for the health of their nation and determine whether they believe that new antibacterial treatments will be required to maintain the health and economic productivity of their workforces.

\section{Sylvie Garneau-Tsodikova}

Q What do you believe is the major hurdle in addressing antibiotic resistance?

Patient treatment for bacterial infections is not based on an in-depth understanding of patient history and bacterial strain resistance profile. There are four major hurdles in addressing antibiotic resistance:

- Bacteria evolve faster than we discover/develop novel drug scaffolds;

- New targets for antibiotics need to be identified as the current drug targets have been overtargeted and we cannot trick them anymore;

- The chemical space (shape, size, polarity, etc.) that we utilize is highly limited. We therefore need to expand the chemical space to identify unique antibiotic scaffolds with novel mechanisms of action;

- Patient treatment for bacterial infections is not based on an in-depth understanding of patient history and bacterial strain resistance profile.

Q What action do you propose is needed in order to curb the emergence of antibiotic resistance?

In addition to expanding the chemical space and identifying new antibiotic targets, we will need a global and detailed knowledge of the current resistant bacterial strains that are out there. Patients are being treated without really knowing the resistance profile of the bacteria that infect them and this is highly problematic. Personalized medicine for cancer patients is something that scientists have been thinking about for a long time. At some point, one will need to begin thinking about a better way of deciding on treatment for bacterial infections. How nonantibiotic drugs affect development of bacterial resistance to antibiotics will also need to come to the forefront of the research endeavor.

Q After a hugely prolific antibiotic discovery period between 1940 \& 1962, there is now a dramatic shortage of new classes of antibiotics under development. What do you believe is the reason for this?

In the 1960s, scientists believed that we had won the battle over bacterial infection. Big Pharma therefore decreased their efforts toward antibiotic discovery while bacteria kept on evolving and developing resistance. We woke up to this issue too late and are now trying to catch up on a big mistake that was made about 60 years ago, which was to think that we are smarter than bac- 
teria. It is very sad to say, but antibiotics do not bring as much money as drugs for long-term chronic diseases. Therefore Big Pharma are not as inclined to put money into antibiotic development, especially as the latest antibiotic developed experienced resistance soon after its introduction to the market. Unfortunately, many are trying to cure the big $\mathrm{C}$ (cancer), but at some point, even if we develop a cure for cancer, these patients will likely die of bacterial infections, if we do not have antibiotics that work. This is a serious problem and we need to all work together to make up for the time lost when new antibiotics were not a primary goal for many scientists.

Q Are there any alternative approaches that you believe may yield more promising results, but are currently being overlooked in the discovery of new antimicrobials?

There are a number of alternatives that we need to look into:

- Natural products from unique sources;

- Repurposing compounds that were originally developed for other diseases and that did not make it onto the market;

- Developing compounds that are broad-spectrum (there are huge debates about broad-spectrum vs narrow spectrum. However, some patients [e.g., military personnel] get infected by multiple bacterial strains);

- Antibiotics with more than one mode of actions (this would help alleviate resistance also);

- Antibiotic cocktails.

Q Taking into account that the majority of antibiotics that are in use today do not strictly adhere to the Lipinski rule of five, do you feel that classically used 'rules of thumb', such as this, should be avoided in the discovery \& design of new antibiotics?

It should not be avoided, but it should surely not prevent the development of compounds that do not obey this rule. Natural products rarely obey Lipinski rule of five and they have been the sources of numerous drugs. In this era, one needs to be more open-minded and less restricted in their way of thinking when developing new antibiotics.

Q If you were to create new set of guidelines/rule of thumb aimed at aiding the discovery \& design of novel antimicrobials, what criteria would you include?
My rules would be:

- Target unique or multiple targets/mechanisms of action, as opposed to targeting something that we have been focusing on with the current antibiotics that do not work;

- No mammalian toxicity;

- Compounds need to be active against a large number of clinical isolates resistant to the current antibiotics;

- Minimum inhibitory concentrations should be in the nanomolar range.

Q In recent decades, the development of new antibiotics has failed to keep pace with the emergence of antibiotic resistance, with only four pharmaceutical companies retaining active antimicrobial drug discovery programs. What do you think needs to be done to re-invigorate the search for new antibiotics, particularly among Big Pharma?

It will be very difficult to get the Big Pharma interested again in antibiotics as antibiotics are not the big money makers. I believe that the government should invest more into helping scientists interested in starting small companies focusing on antibiotic discovery/ development, which could then pair with Big Pharma when the products are well along the way. The government should also invest in scientists in academia and help them start companies and/or team up with Big Pharma.

Q To what extent do you believe governments worldwide can help stem the rise of antibiotic resistance \& stimulate research toward new drugs?

The governments worldwide are the only ones that can help stimulate research toward new antibiotics. I know that they are now trying, but they are still not putting enough money toward this research area. Not only do individual countries need to provide money to their scientists to develop new antibiotics, but more importantly, joint grant applications between countries will be required to get this problem solved. If we do not unite, we will lose this battle quickly. We all face the same problem and we all need to work together to solve it. One country cannot do it on its own. We can all learn a lot from each other. It is important for the generation that is growing up to learn to collaborate and experience what other people live with every day to understand the immensity of the problem at hand and how to better solve it. 


\section{Colin Garner}

\section{Q What action do you propose is needed} in order to curb the emergence of antibiotic resistance?

There needs to be a greater awareness of the problem and how overuse of antibiotics in both veterinary and human medicine has contributed to antibiotic resistance. For example, one of Antibiotic Research UK's (ANTRUK) goals is to educate the public and professionals about antibiotic resistance to make sure that antibiotics are only used when necessary. However much more needs to be done to control and prevent infection. I am always surprised when I visit some hospitals that medical staff in their uniforms are mingling with the general public with the possibility of spreading infection. Readers may not be aware that if patients are put in a room or ward that has previously held a patient with an antibiotic-resistant infection, that the subsequent patient is more likely to contract an antibiotic-resistant infection because of insufficient room cleaning and dsinfection.

Q Studies indicate that the issue of antibiotic resistance is particularly high in the developing world \& in the emerging economies known as the 'BRIC' states. Why do you believe this is the case?

This is often associated with easy access to antibiotics which are often available OTC without a prescription.

Q After a hugely prolific antibiotic discovery period between 1940 \& 1962, there is now a dramatic shortage of new classes of antibiotics under development. What do you believe is the reason for this?

A lack of funding and a lack of ideas. Until the reimbursement model for antibiotics is changed there is no incentive for pharma companies to invest in research. Antibiotic treatment courses are short in comparison with, for example, diabetes treatments. Companies will always focus on developing drugs with high volume sales potential. If a new antibiotic is discovered for the treatment of antibiotic resistance, to safeguard against resistance to the new drug, it should be used as little as possible, in other words, sit on the pharmacy shelf and only be used occasionally. A new method of reimbursement needs to be found and found soon. The funding of basic science of antibiotic resistance needs also to be substantially increased to find the new antibiotics of the future.

\section{Q Target-based screening using knowledge of} bacterial genomics has received a great deal of attention in the last decade, yet has so far proven unsuccessful in bringing new antimicrobials to the market. What do you think is the reason for this \& do you feel it is time to begin focusing on alternative approaches in the discovery of new antimicrobials?

Bacteria have been on this planet for 3.5 billion years because they can adapt to new environments very quickly. They have multiple pathways to fight off toxins of which an antibiotic is just one. The molecular targeting approach fails to take into account these diverse pathways. We need to go back to basics and focus much more on phenotypic screens such as those being conducted by ANTRUK. To give two examples: an interesting area to explore is to seek antibiotic resistance breakers (ARB), that is drugs or molecules which are not antibiotics per se but in combination with an antibiotic can overcome resistance and very little research seems to have been conducted on using antibiotic combinations in the same way that combinations are routinely used for cancer treatment.

\section{Q Are there any alternative approaches that} you believe may yield more promising results, but are currently being overlooked in the discovery of new antimicrobials?

There are a multitude of approaches that could be examined such as ARB, vaccines, monoclonal antibodies, phage, host cell immunity, natural products, etc., in addition to the classical small molecules. Many of these are under examination currently but it could be at least 10 years before any of these approaches result in marketable drugs.

Q Taking into account that the majority of antibiotics that are in use today do not strictly adhere to the Lipinski rule of five, do you feel that classically used 'rules of thumb', such as this, should be avoided in the discovery \& design of new antibiotics?

Rules of thumb are just that rules of thumb. Phenotypic screening approaches should be revived and antibiotic-resistant bacteria should be the target.

\section{Q If you were to create new set of} guidelines/rule of thumb aimed at aiding the discovery \& design of novel antimicrobials, what criteria would you include?

Nothing should be ruled out but in terms of finding new chemical entities (NCEs) it will be more productive to look at existing chemical scaffolds rather than to find new ones.

\section{Q It has been argued that if penicillin was} discovered today, it would never have made it to the market due to regulatory restrictions. With this in mind, do you feel that current regulations stifle the development of novel antibiotics? 
In all probability, yes. In particular, with regards to clinical trial regulatory requirements, which although have been revised still require further revisions. Bayesian study designs need to be used and we need to get much more quickly from our in vitro findings to humans. This can be facilitated by the use of Phase 0 clinical studies which can provide quick and cheap information about pharmacokinetic/pharmacodynamics properties.

Q In recent decades, the development of new antibiotics has failed to keep pace with the emergence of antibiotic resistance, with only four pharmaceutical companies retaining active antimicrobial drug discovery programs. What do you think needs to be done to re-invigorate the search for new antibiotics, particularly among Big Pharma?

There needs to both financial R\&D push and marketing pull. The existing model of volume-driven sales is inappropriate for new antibiotics as one wants to avoid the emergence of resistant strains, which means that often the drug will sit on the shelf and only be used as a drug of last resort when our existing antibiotics have failed. In addition an antibiotic course is short compared with a drug to treat cancer or heart disease. New financial models as proposed by the O'Neill review need to be considered [4]. The UK has highlighted the problem of antibiotic resistance and needs to take first leader action. This requires new forms of finance to be looked at for R\&D, clinical trials and postmarketing.

Q Do you feel that there is a need for a new commercial model that would help incentivize Big Pharma to invest in antimicrobial discovery? Yes, but which model is a matter of debate. In the US legislation, for example, the GAIN Act has been introduced but this does not appear to have accelerated the development of new antibiotics. The Act offers a 5-year patent extension on new antibiotics but if the volumedriven sales model is no longer apposite then it is hard to see what benefits the Act provides.

Q Bearing in mind that the issue of antimicrobial resistance is more acute in developing countries, how can it be ensured that new antimicrobials are priced at a level that is both commercially attractive to pharmaceutical companies \& is also accessible to the developing world?

As a charity, the issue of price is less important to us than finding new antibiotic therapies. Provided we can cover our costs and that we can work with like-minded organizations such as Wellcome Trust (London, UK), Bill \& Melinda Gates Foundation (Seattle, WA, USA), etc. then it should be possible to make new therapies available to the poorest of the poor. In order to avoid the overuse and lack of controls to any new therapies we might develop our charity is focusing on intravenously administered drugs which could only be given by medical practitioners.

Q The process of bringing new drugs from the bench to the bedside is on average 10-15 years. With this in mind, do you feel that investing in the discovery of innovative new drugs is a promising solution to the antibiotic crisis? Yes and no. We believe if ARB to our existing antibiotics can be found then this could substantially shorten drug development times allowing our existing antibiotics to continue to work while new ones are developed. We estimate it will take 5-7 years to get an ARB registered for clinical use at a cost of no more than $£ 30$ million. This approach should be cheaper and faster than traditional approaches because ARBs are not NCE but existing drugs and so drug development costs should be substantially less than for an NCE.

Q How do you anticipate the field of antimicrobial drug discovery progressing over the next

5-10 years?

I am optimistic that approaches such as those taken by ANTRUK and others will lead to new therapies being available. This will however be dependent on a big increase in funding, better career prospects for young scientists, good science, incentivizing pharma and generally increasing the profile of this severely under-funded area.

\section{Financial \& competing interests disclosure}

The authors have no relevant affiliations or financial involvement with any organization or entity with a financial interest in or financial conflict with the subject matter or materials discussed in the manuscript. This includes employment, consultancies, honoraria, stock ownership or options, expert testimony, grants or patents received or pending, or royalties.

No writing assistance was utilized in the production of this manuscript.

\section{References}

1 Takrouri K, Cooper HD, Spaulding A et al. Progress against Escherichia coli with the oxazolidinone class of antibacterials: test case for a general approach to improving whole-cell gramnegative activity. doi:10.1021/acsinfecdis.6b00003 (2016) (Epub Ahead of Print).

2 Antibiotic Action. http://antibiotic-action.com

3 Antimicrobial stewardship: managing ANTIBIOTIC Resistance. www.futurelearn.com/courses

4 Review On Antimicrobial Resistance. Securing New Drugs For Future Generations: The Pipeline Of Antibiotics. http://amr-review.org/sites/default/files/ 